\title{
Identification of candidate genes and pathways in dexmedetomidine-induced neuroprotection in rats using RNA sequencing and bioinformatics analysis
}

\author{
Li Yang ${ }^{1,2 \#}$, Haiying Wu ${ }^{2 \#}$, Fanglin Yang ${ }^{1,2},{\text { Ping } \mathrm{Li}^{3} \text {, Yongjie Huang }}^{2}$, Xinyue Zhang ${ }^{1,4}$, Chuanyun Qian ${ }^{2}$ \\ ${ }^{1}$ Kunming Medical University, Kunming, China; ${ }^{2}$ Emergency Department, the First Affiliated Hospital of Kunming Medical University, Kunming, \\ China; ${ }^{3}$ Department of Anatomy and Histology/Embryology, Faculty of Basic Medical Sciences, Kunming Medical University, Kunming, China; \\ ${ }^{4}$ Department of Geriatric Neurology, the First Affiliated Hospital of Kunming Medical University, Kunming, China \\ Contributions: (I) Conception and design: H Wu, C Qian, L Yang; (II) Administrative support: C Qian, H Wu, P Li; (III) Provision of study materials \\ or patients: L Yang, P Li; (IV) Collection and assembly of data: L Yang, F Yang, Y Huang, X Zhang; (IV) Data analysis and interpretation: L Yang, F \\ Yang, Y Huang; (VI) Manuscript writing: All authors; (VII) Final approval of manuscript: All authors. \\ "These authors contributed equally to this work. \\ Correspondence to: Chuanyun Qian. Emergency Department, the First Affiliated Hospital of Kunming Medical University, No. 295 Xichang Road, \\ Kunming, China. Email: qianchuanyun@126.com.
}

Background Traumatic brain injury (TBI) is a major cause of disability worldwide, without definitive and effective intervention. Dexmedetomidine (DEX) has a neuroprotective effect against TBI; however, the detailed mechanism underlying this effect remains unclear.

Methods: Ten male Sprague Dawley rats were used to establish a TBI model. The rats were randomly divided into two groups: the TBI group (TBI, control group) and the DEX treatment group (DEX). The next day, the neurological function of the rats were evaluated by the modified neurological severity score (mNSS). Then, the rats were sacrificed, and RNA sequencing was performed to identify differentially expressed messenger RNAs (mRNAs) and microRNAs (miRNAs) in brain tissue samples. Additionally, we performed a bioinformatics analysis to explore the candidate genes and pathways that might play important roles in DEX-induced neuroprotection. The most significantly differentially expressed miRNAs and possible hub genes were validated by quantitate reverse transcription-polymerase chain reaction (qRT-PCR) using more samples.

Results: In the DEX group, 517 mRNAs (352 up-regulated and 165 down-regulated) and 35 miRNAs (18 up-regulated and 17 down-regulated) were differentially expressed compared to the TBI group. Gene Ontology analysis revealed the up-regulated mRNAs to be significantly enriched in microtubule-based movement or processes, microtubule and tubulin binding. Kyoto Encyclopedia of Genes and Genomes analysis showed that these up-regulated mRNAs were significantly enriched in the B-cell receptor signaling pathway as well as the cell cycle pathway. Also, Lyn and Cdk1 were found to be associated with the B-cell receptor signaling and cell cycle pathways, respectively. Furthermore, the down-regulated miRNAs were significantly enriched in cellular components, although no significant Gene Ontology terms or KEGG pathways were found for the down-regulated mRNAs or up-regulated miRNAs.

Conclusions: Differentially expressed mRNAs and miRNAs were identified after the administration of DEX in a TBI rat model. The B-cell receptor signaling pathway and the cell cycle pathway might be involved in the neuroprotective effect of DEX against TBI, Lyn and Cdk1 might be hub genes.

Keywords: Traumatic brain injury (TBI); dexmedetomidine (DEX); RNA sequencing; bioinformatics analysis

Submitted Oct 28, 2020. Accepted for publication Jan 02, 2021.

doi: 10.21037/apm-20-2346

View this article at: http://dx.doi.org/10.21037/apm-20-2346 


\section{Introduction}

Traumatic brain injury (TBI) is a leading cause of disability globally. It is reported that approximately 10 million people suffer TBI annually, which places a significant heavy load on public health $(1,2)$. TBI negatively impacts individuals' life quality and brings a heavy family and societal burden (3). Because of the immediate and delayed effects of injury, the pathology of TBI is not merely complex, but is heterogeneous (4). The neuropathology of TBI comprises primary and secondary injury. The primary injury results from traumatic insult and the direct effects of mechanical forces (5). The secondary injury is caused by the cascade of molecular events and cytopathic reactions triggered by the primary injury, including brain edema, hypoxicischemic injury, metabolic disturbance, vascular injury, and inflammation, which aggravate the neuropathology of TBI $(5,6)$. Further insight into the neuropathy of TBI and the mechanism underlying secondary brain injury is crucial to developing novel and definite clinical interventions.

Dexmedetomidine (DEX), a highly selective $\alpha-2$ adrenergic receptor $(\alpha-2 \mathrm{AR})$ agonist, is widely used in clinical anesthesia and the intensive care unit (ICU). It has been confirmed that DEX has a protective effect on multiple organs, such as the nervous system, lungs, heart, kidneys, and liver (7); among them, DEX exerts its earliest and deepest effects on the nervous system. DEX has been demonstrated to protect against cerebral hypoxia-ischemia injury and lipopolysaccharide-induced neuroinflammation $(8-10)$, as well as against hyperoxia-induced toxicity in the brains of neonatal rats $(11,12)$. It has also been shown to improve nervous system function after brain injury (13), to attenuate anesthetic toxicity in developing neurons $(14,15)$, and to reduce the incidence of postoperative delirium and cognitive impairment (16). In vivo and in vitro studies of TBI models have also revealed that DEX exhibits a neuronprotective effect $(17,18)$. However, the exact molecular mechanisms of the positive and protective characteristics of DEX are not completely understood. In the future, more efforts are needed to validate the exact mechanism through which DEX exerts organ protective effects.

Along with the development of transcriptomic analysis, which is a widely used genomic analysis technique that uses microarrays or RNA sequencing to quantify global RNA expression, molecular diagnosis has been employed to characterize specific pathologic states following TBI (19). However, transcriptomic changes associated with neuroprotective agents have not yet been reported.
A number of studies have used high-throughput tools to explore diagnostic or therapeutic targets for TBI, and some progress has already been made $(20,21)$. Bioinformatics is an emerging discipline that developed following the launch of the Human Genome Project (22); it has since become one of the most fundamental research methods in the life sciences. Bioinformatics analysis enables the mining and analysis of massive data sets, as well as the exploration of key genes and pathways associated with particular diseases. To better understand the exact mechanism of the neuroprotective effect of DEX and to discover new potential therapeutic targets for TBI, we aimed to identify the messenger RNAs (mRNAs) and microRNAs (miRNAs) that are differentially expressed after DEX administration in rat brains by performing RNA sequencing and bioinformatics analysis. We also aimed to determine candidate genes and signaling pathways that might play considerable roles in the neuroprotective effect of DEX against TBI. We present the following article in accordance with the ARRIVE reporting checklist (available at http://dx.doi.org/10.21037/apm-202346).

\section{Methods}

\section{Animals}

Healthy male Sprague Dawley rats of $10 \pm 2$ weeks (weight $300 \pm 20 \mathrm{~g}$ ) were used in our study. The rats were housed in specific-pathogen-free conditions in our laboratory and given free access to food and water. The rats were kept at $22-24{ }^{\circ} \mathrm{C}$ with a 12 -hour light/12-hour dark cycle. Animal experiment protocols were approved by the Ethics Review Committee for Laboratory Animals of Kunming Medical University (Approval no. KMMU2020161). Animals were treated in accordance with Guide for the Care and Use of Laboratory Animals (8th edition, National Academies Press).

\section{TBI model}

A rat model of TBI was established using the modified Feeney's weight-drop method (23). The rats were anesthetized with $2 \%$ sevoflurane and $3 \%$ pentobarbital sodium $(30 \mathrm{mg} / \mathrm{kg}$, i.p.). First, in order to expose the skull, a midline incision was made along the central cranial line. Then, a small window ( $5 \mathrm{~mm}$ in diameter) was made at $3 \mathrm{~mm}$ to the right of the coronal suture and $3 \mathrm{~mm}$ behind the sagittal suture with an orthopedic drill, keeping the dura 
intact. Afterward, a 40-g weight was dropped from a height of $25 \mathrm{~cm}$ onto the exposed dura, resulting in a $3-\mathrm{mm}$-deep wound on the brain (24).

\section{Experimental protocols}

DEX (Hengrui Pharmaceutical Co., Ltd, Jiangsu, China) was dissolved in normal saline (NS) and administered via intraperitoneal injection. Based on our previous study (25) and according to the manufacturer's recommendations, a DEX dose of $100 \mu \mathrm{g} / \mathrm{kg}$ was finally selected in the present study.

The rats were randomly divided into two groups: the TBI group ( $\mathrm{n}=5)$, which was administered NS with the same volume as DEX group 1 hour after modeling; and the DEX group ( $\mathrm{n}=5)$, which was administered DEX intraperitoneally at a dose of $100 \mu \mathrm{g} / \mathrm{kg} 1$ hour after modeling. After 24 hours, all experimental animals were sacrificed.

\section{Modified neurological severity score (mNSS)}

mNSS of the rats was evaluated 24 hours after modeling by who was not aware of the study design, Scoring is based on tail-lifting, walking, sensory, balance beam, loss of reflex, and abnormal movement tests. The maximum score is 18 points, and the score is positively correlated with the degree of injury. All the experimental rats underwent mNSS evaluation, but only three rats from each group were selected for subsequent test.

\section{Total RNA isolation}

Total RNA was isolated from the damaged brain issue of each rat using TRIzol reagent (MRCGUER, Co., Inc., Germany) according to the manufacturer's instruction. The purity and integrity of the RNA were assessed using the Nano Photometer spectrophotometer (IMPLEN, CA, USA) and the RNA Nano 6000 Assay Kit of the Bioanalyzer 2100 system (Agilent Technologies, CA, USA). The ration of the samples' absorbance at 260 and $280 \mathrm{~nm}$ (A260/ A280) $>1.8$ and an RNA integrity number (RIN) $>7.0$ were considered to show adequate purity for further analysis.

\section{RNA sequencing}

After the extraction of total RNA, the quantification and qualification, library preparation, and subsequent RNA sequencing of samples were performed by Novogene Co.,
Ltd. (Beijing, China). The edge RR package (version 3.18.1) was used to analyze the differentially expressed mRNAs and miRNAs between the TBI group and the DEX group. $P$ values were adjusted using the Benjamini-Hochberg method, and an adjusted $P$ value of 0.05 was set as the threshold for significantly differential expression.

\section{Prediction and selection the target genes of differentially expressed miRNAs}

The target genes of differentially expressed miRNAs were computationally predicted with miRWalk 2.0 (http://zmf. umm.uni-heidelberg.de/apps/zmf/mirwalk2/miRretsys-self. html) (26) using miRanda and RNAhybrid. Since the main function of miRNA is to inhibit the transcription of mRNA, the predicted target genes were compared with the mRNA sequencing and miRNA sequencing data, and only target genes that were inversely correlated with the differentially expressed miRNAs were selected as target genes.

\section{Gene Ontology (GO) and Kyoto Encyclopedia of Genes and Genomes (KEGG) pathway analysis}

The Database for Annotation, Visualization and Integrated Discovery (DAVID; http://david.ncifcrf.gov) (27) (version 6.7) was used for GO analysis and KEGG pathway analysis. The significant GO terms and KEGG pathways were defined as those with a corrected $\mathrm{P}$ value $<0.05$ and number of enriched genes $\geq 1$.

\section{Construction of a Protein-Protein Interaction (PPI) network}

The Search Tool for the Retrieval of Interacting Genes/ Proteins (STRING) version 10.5 (https://string-db. org/) (28) was used to construct a PPI network in order to shed light on the functional associations between the transcription products of the differentially expressed genes. Proteins with an interaction score $>0.4$ were considered to be statistically significant. Genes with a connectivity degree of $\geq 10$ were selected as hub genes. Cytoscape software version 3.7.0 (http://cytoscape.org), an open-source bioinformatics software platform (29), was used to visualize molecular interaction networks.

\section{Construction of a miRNA/mRNA integrated network}

To further clarify the interactions between miRNAs 
Table 1 Primers used for quantitative real-time reverse transcription-polymerase chain reaction

\begin{tabular}{ll}
\hline Factors & Primers \\
\hline Cdk1 & Forward: TCAAGTGGTAGCCATGAAAAAAA \\
& Reverse: ATAACCTGGAATCCTGCATAAGC \\
Lyn & Forward: ATGGGATGTATTAAATCAAAAAGGA \\
& Reverse: AAAGTTGAGATTCAGGAACTGGC \\
GAPDH & Forward: GAGTCAACGGATTTGGTCGT \\
& Reverse: GACAAGCTTCCCGTTCTCAG \\
miR-7a-5p & Forward: TGGAAGACTAGTGATTTTTTGT \\
miR-873-5p & Forward: TGATTGTCCAAACGCAATTCT \\
miR-135a-3p & Forward: TGTAGGGATGGAAGCCATGAAA \\
\hline
\end{tabular}

and mRNAs, a miRNA/mRNA integrated network was constructed using the differentially expressed miRNAs and the selected target genes. The integrated network was visualized by using Cytoscape soft version 3.7.0 (http:// cytoscape.org) (29).

\section{Quantitative real-time reverse transcription-polymerase chain reaction ( $q$ RT-PCR)}

Total RNA was extracted using TRIzol reagent (MRCGUER, Co., Inc., Germany) following the manufacturer's protocol. Reverse transcription was performed using a SureScript ${ }^{\mathrm{TM}}$ First-Strand cDNA Synthesis Kit (GeneCopoeia, America). The primers for miR-7a-5p, miR-873-5p, miR-135a-3p, CDK1, Lyn, and glyceraldehyde 3-phosphate dehydrogenase (GAPDH) were designed and synthesized by TSINGKE Biological Technology, Ltd. (China). qRT-PCR was performed following the instructions supplied with the PCR kit (GeneCopoeia, America), with GAPDH used as an internal reference. The fold changes were calculated by means of relative quantification $\left(2^{-\Delta \Delta \mathrm{Ct}}\right.$ method). The primers used in our study are presented in Table 1 .

\section{Statistical analysis}

Statistical analyses were performed using GraphPad Prism 8.0 (GraphPad Software, La Jolla, CA, USA). Data are shown as mean \pm standard error of the mean (SEM). Student's $t$-test was used to compare two independent groups. A corrected $\mathrm{P}$ value $<0.05$ was considered to be statistically significant.

\section{Results}

\section{DEX administration following TBI exerted a neuroprotective effect in vivo}

To confirm the neuroprotective effect of DEX, an in vivo rat model of TBI was established (Figure 1A). Behavioral changes of the TBI rats were evaluated using the mNSS ( $\mathrm{n}=5$ for each group). Our results showed that the mNSS in the DEX group was significantly lower than that in the TBI group (Figure 1B) (30); this is similar to the findings of $\mathrm{Li}$ et al.'s study, in which DEX was associated with increased behavioral function. Subsequently, to further explore the neuroprotective mechanism of DEX, a multi-step protocol was applied to analyze the biological functions and potential roles of these deregulated mRNAs and miRNAs ( $\mathrm{n}=3 / 5$ for each group) (Figure 1C).

\section{DEX induced changes in mRNA expression profile in rat brain}

To explore the potential biotargets involved in the neuroprotective effect of DEX against TBI, differentially expressed mRNAs were identified in the injured area of the ipsilateral hemisphere cerebral cortex of rats (Figure 2A). A total of 517 mRNAs were found to be differentially expressed in the DEX group compared to the TBI group, including 352 that were up-regulated and 165 that were down-regulated (Tables S1,S2). A hierarchical heat map was created to show the expression levels of these deregulated mRNAs (Figure 2B). We further identified the differentially expressed mRNAs with a fold change $>2 ; 125$ differentially expressed mRNAs had a fold change $>2$ (70 up-regulated and 55 down-regulated), and these mRNAs are presented in a volcano plot (Figure 2C).

\section{Lyn and Cdk1 may be the potential targets of DEX}

To understand the possible functions of these potential biotargets, GO and KEGG pathway enrichment analyses and a PPI network analysis were conducted. GO analysis of the up-regulated mRNAs revealed numerous significantly enriched biological terms (Figure 2D), including subcellular components, microtubule-based movement or processes, microtubule motor activity, and microtubule and tubulin binding, as well as cytoskeletal protein binding, which are reportedly associated with the neuronal microenvironment and neuropathological mechanotransduction in TBI (31). 
A

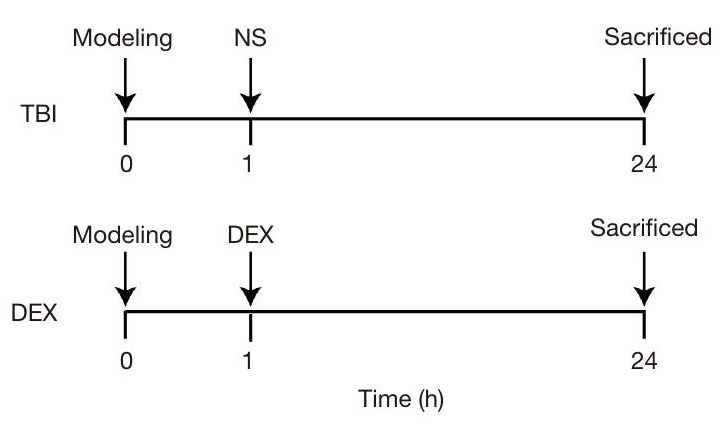

B

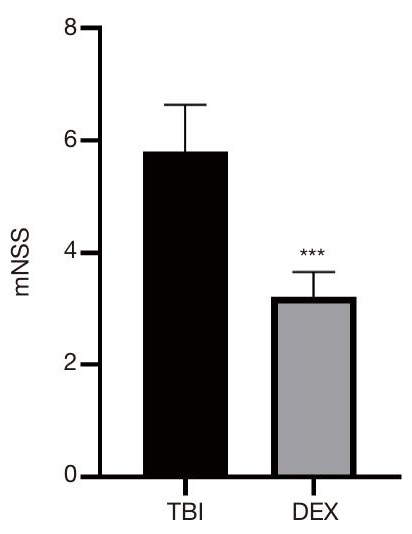

C

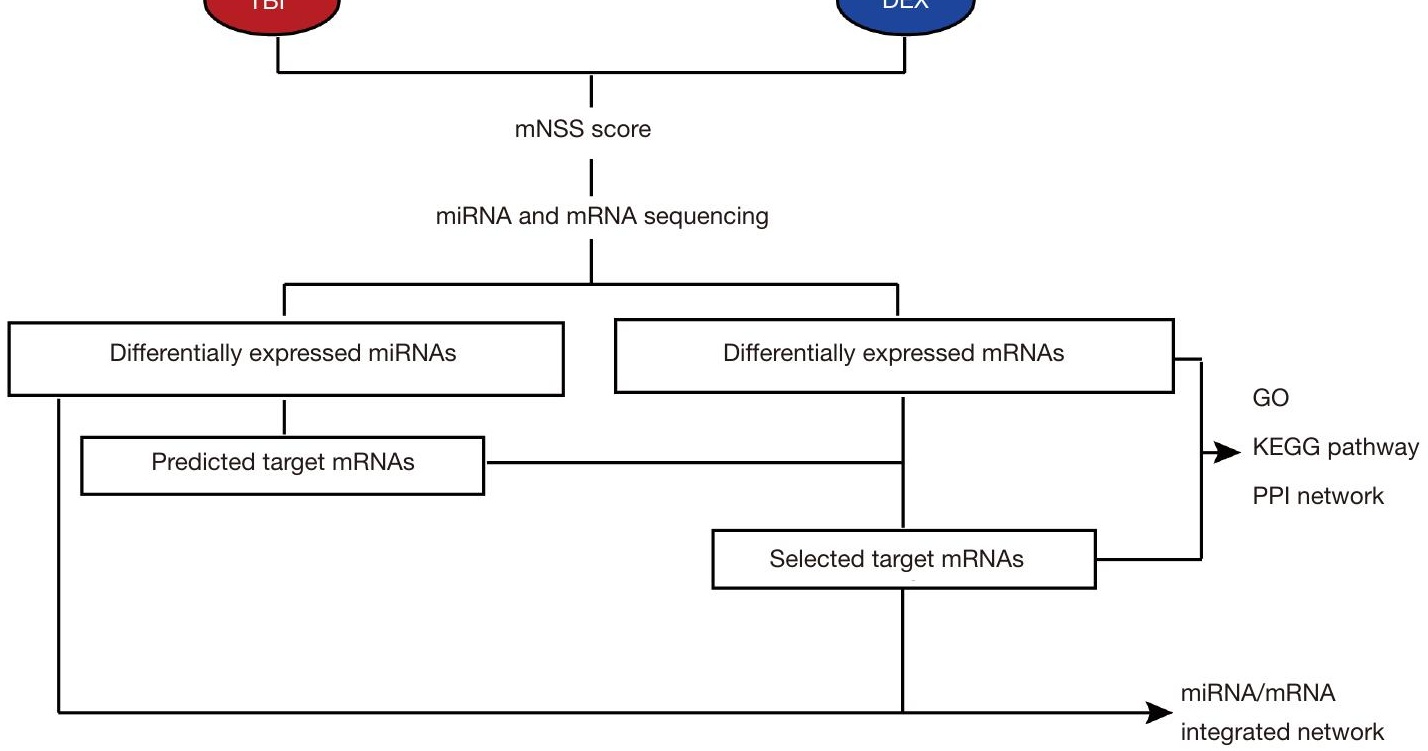

Figure 1 DEX administration after TBI exerts neuroprotective effects in vivo. (A) Experimental protocol. (B) DEX administration after TBI was associated with a lower mNSS. (C) Multi-step approach for analysis of the differentially expressed mRNAs and miRNAs. ${ }^{* * *} \mathrm{P}<0.001$ vs. TBI. N=5 in each group. TBI, traumatic brain injury; DEX, dexmedetomidine; mNSS, modified neurological severity score; GO, Gene Ontology; KEGG, Kyoto Encyclopedia of Genes and Genomes; PPI, protein-protein interaction.

Further, in the KEGG pathway analysis, the cell adhesion molecule, cell cycle, and B-cell receptor signaling pathways were found to be significantly enriched (Figure 2D). However, for the down-regulated mRNAs, no significant GO terms or KEGG pathways were detected. The PPI network analysis of the up-regulated mRNAs revealed 25 hub genes (Figure 2E), including Lyn and Cdk1, which have been reported as being largely related to the B-cell receptor signaling and cell cycle pathways, and the $C d k$ family has proved to be a potential target for the treatment of a variety of neurological diseases $(32,33)$.

\section{DEX induced changes in miRNA expression profile in rat brain}

The miRNA sequencing revealed 35 differentially expressed 


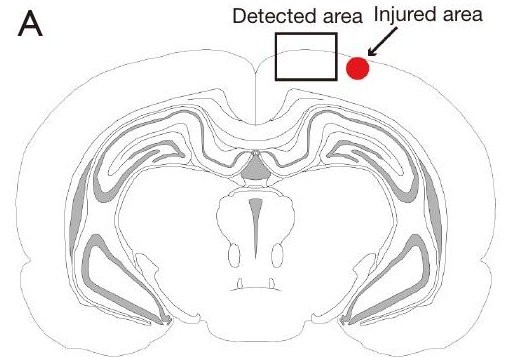

C

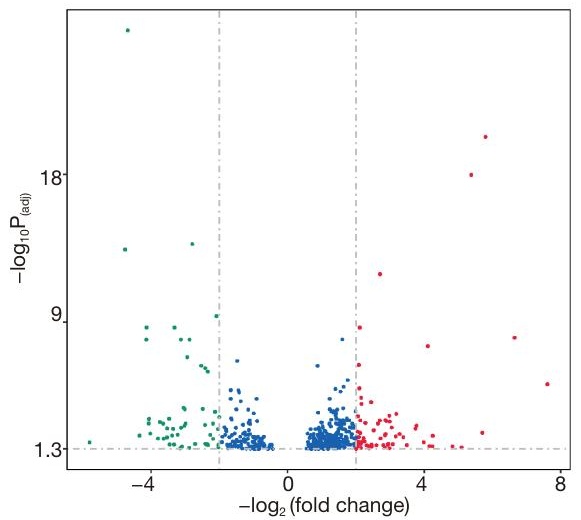

$\mathrm{D}$

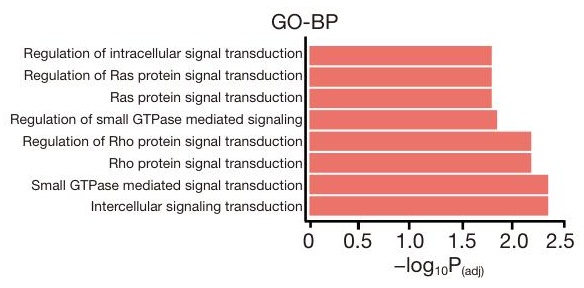

GO-MF
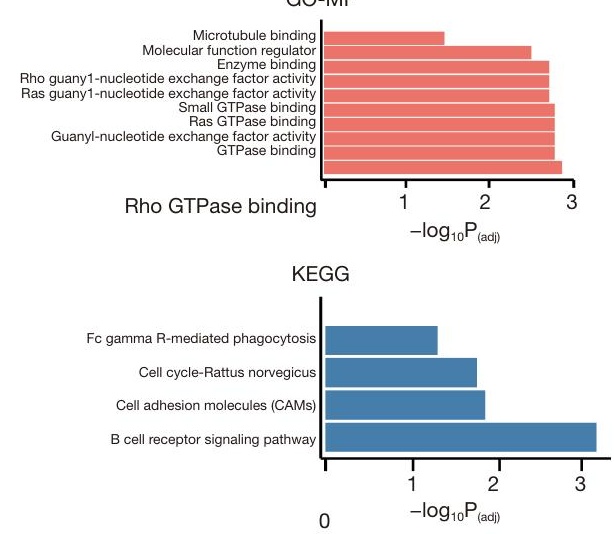

B

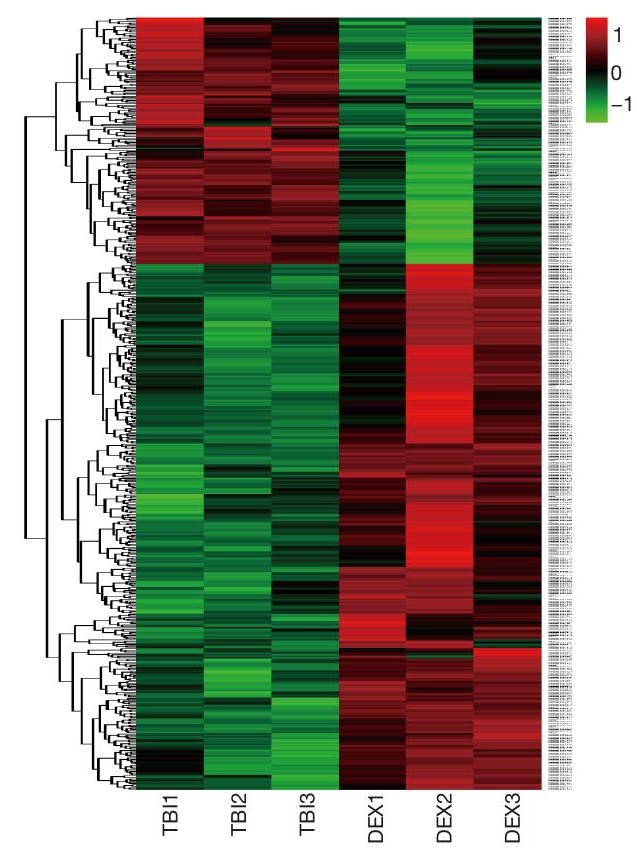

$\mathrm{E}$

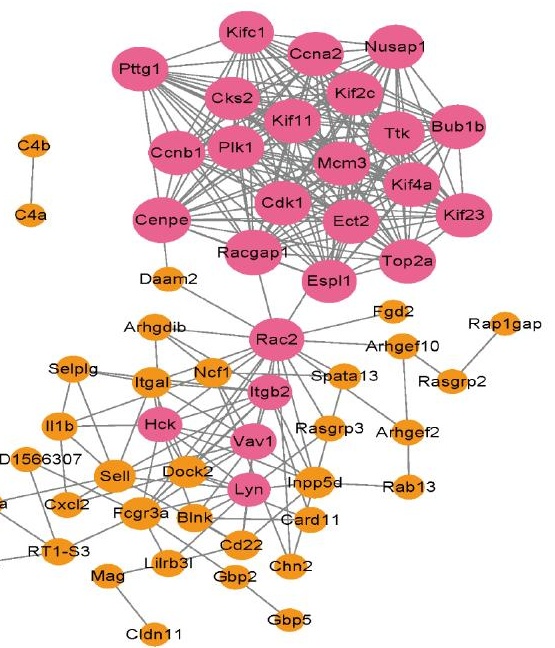

Figure 2 Bioinformatics analysis of the deregulated mRNAs suggested that $L y n$ and $C d k 1$ may be potential targets of DEX. (A) Schematic diagram of the sampling site. (B) Heat map of differentially expressed mRNAs after DEX administration (red: up-regulated genes, green: down-regulated genes). (C) Volcano plot of differentially expressed mRNAs with a fold change $>2$ after DEX administration (red represents up-regulated genes, and green represents down-regulated genes). (D) GO and KEGG pathway analyses of the up-regulated mRNAs. (E) PPI network analysis of the up-regulated mRNAs (orange nodes indicate up-regulation, and pink nodes represent hub genes. The edges represent the relationships between genes). $\mathrm{N}=3$ in each group. DEX, dexmedetomidine; BP, biological process; MF, molecular function; GO, Gene Ontology; KEGG, Kyoto Encyclopedia of Genes and Genomes; PPI, protein-protein interaction. 
miRNAs (18 up-regulated and 17 down-regulated) between the TBI group and the DEX group (Tables S3 and S4). These differentially expressed miRNAs are shown in a hierarchical heat map and a volcano plot (Figure $3 A$ and $B$, respectively). To establish the exact role of these deregulated miRNAs in DEX-induced neuroprotection, bioinformatics analysis methods similar to those used for mRNAs were used. We found GO term with intercellular part was significantly involved in the neuroprotective effect (Figure 3C), which is consistent with the findings of previous studies that confirmed DEX to protect neurons (34), microglial cells (35), and astrocytes (36) in virous pathological conditions. However, for the up-regulated miRNAs, no significant GO terms or KEGG pathways were detected. Considering the small number of deregulated miRNAs, further bioinformatics analysis was not performed. More follow-up studies are needed to detect the exact role of miRNAs in the neuroprotective effect of DEX against TBI.

\section{Bioinformatics analysis of the differentially expressed miRNAs and their selected target genes}

To further verify the potential effective biotargets of DEX, the predicted target genes of the miRNAs that were differentially expressed between the two groups were explored using the Novomagic, a free online platform for data analysis (https://magic.novogene.com). Our preliminary screening found that the 18 up-regulated miRNAs and 17 down-regulated miRNAs had 941 and 1,161 target genes, respectively (Table S5 and S6). Then, considering the fundamental biological functions of miRNAs, only the target genes that were negatively regulated by the differentially expressed miRNAs were selected for further analyses. Finally, 23 and 6 target genes were obtained for the down-regulated and up-regulated miRNAs, respectively (Tables S7 and S8), and were further studied. Analyses of the six target genes of the up-regulated miRNAs failed to obtain any significantly enriched GO terms or KEGG pathways, which may be due to the relatively small number of target genes. Considering the six selected target genes are extremely unlikely to play an essential role in DEX-induced neuroprotection, miRNA/ mRNA integrated analysis was not performed.

Analyses of the 23 selected target genes of the downregulated miRNAs revealed that several GO terms were specifically enriched, including the negative regulation of small GTPase-mediated signal transduction, which has emerged as a central process in the molecular pathogenesis of glioblastoma (37), and the Golgi apparatus (Figure 3D). However, we failed to obtain an enriched KEGG pathway of significance using the 23 selected genes. No hub genes or mRNAs regulated by several miRNAs were detected by the miRNA/mRNA integrated network analysis. However, the PPI network analysis uncovered multiple significant connections between proteins, which require further study (Figure 3E).

\section{Validation of the results by qRT-PCR}

The two hub genes mentioned above, Lyn and Cdk1, and the top three differentially expressed down-regulated miRNAs were validated by qRT-PCR. Using more experimental animals ( $\mathrm{n}=5$ in each group), we confirmed that $C d k 1$ was stably up-regulated, while miR-7a-5p and miR-873-5p were firmly down-regulated in validated tests (Figure 4), suggesting that these factors deserve further study. However, the expression patterns of Lyn and miR$135 \mathrm{a}-3 \mathrm{p}$ in validated analysis were the opposite to those shown in the results of RNA sequencing.

\section{Discussion}

Despite the large number of preclinical and clinical studies that have been performed, intervention strategies for TBI remain a problem, due to the complex, heterogeneous pathological changes of this condition. There is no definitive therapy that has been proven to reduce longterm cognitive impairment, and options for rehabilitation are limited $(38,39)$. Traditionally, DEX has been regarded as a neuroprotective agent against TBI, but the exact mechanism has remained unclear. With the development of basic research, growing and consistent preclinical evidence has identified DEX as an effective sedative agent that is less neurotoxic to the developing brain, which also possesses neuroprotective properties in neonatal and other settings of ongoing acute neurological injury (40), including neurosurgical patients (34), ischemic brain injury (41), and TBI. The emerging genomic "bench-to-bed" technique that advances discoveries of cellular biomarkers and mechanisms from animal or cell models through to clinical application, may provide a useful alternative method for identifying potential targets of DEX. We used transcriptomic and bioinformatics analysis, and identified some potential key genes and pathways that might play important roles in the neuroprotective effect of DEX after TBI.

We identified 517 differentially expressed mRNAs, 
A
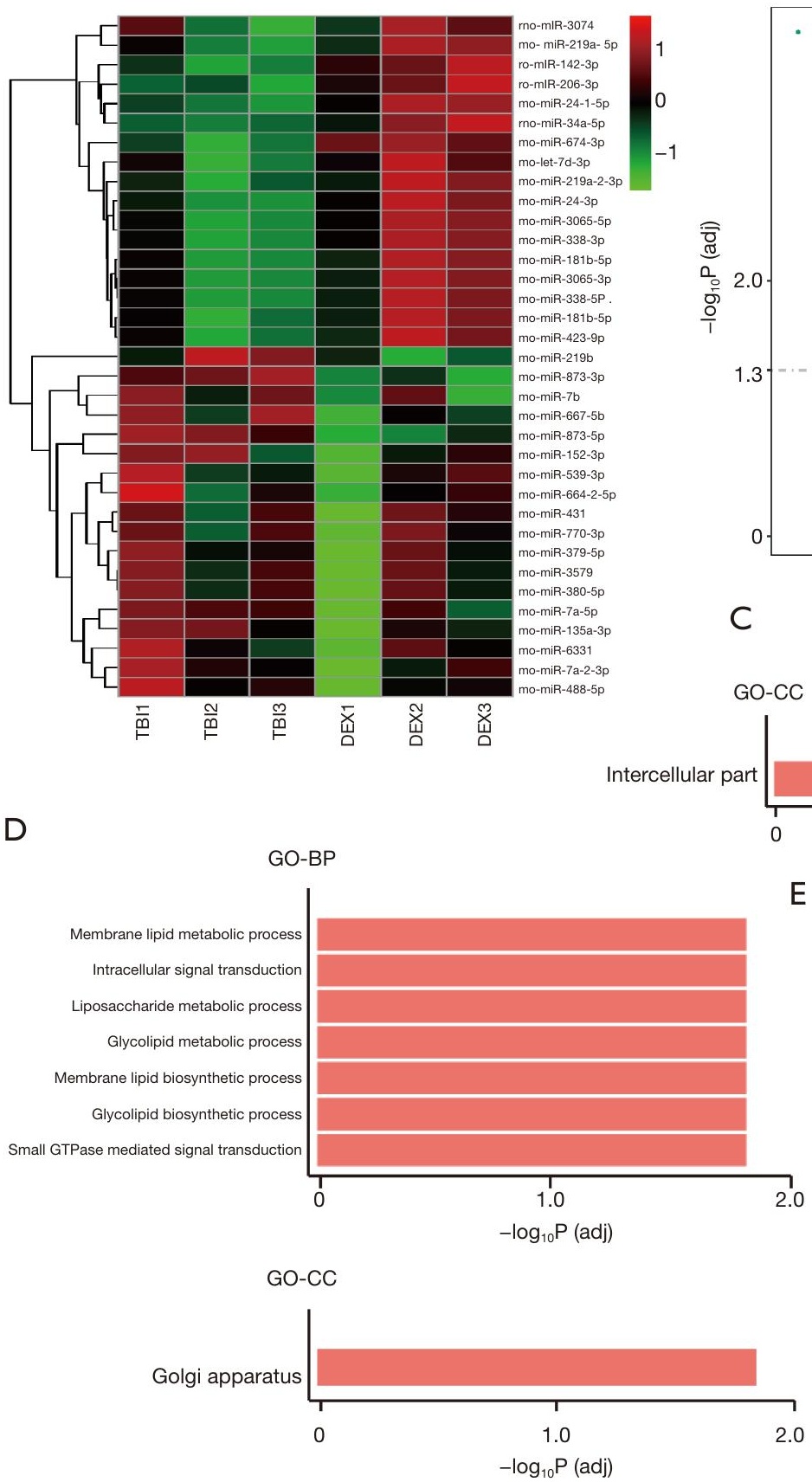

B

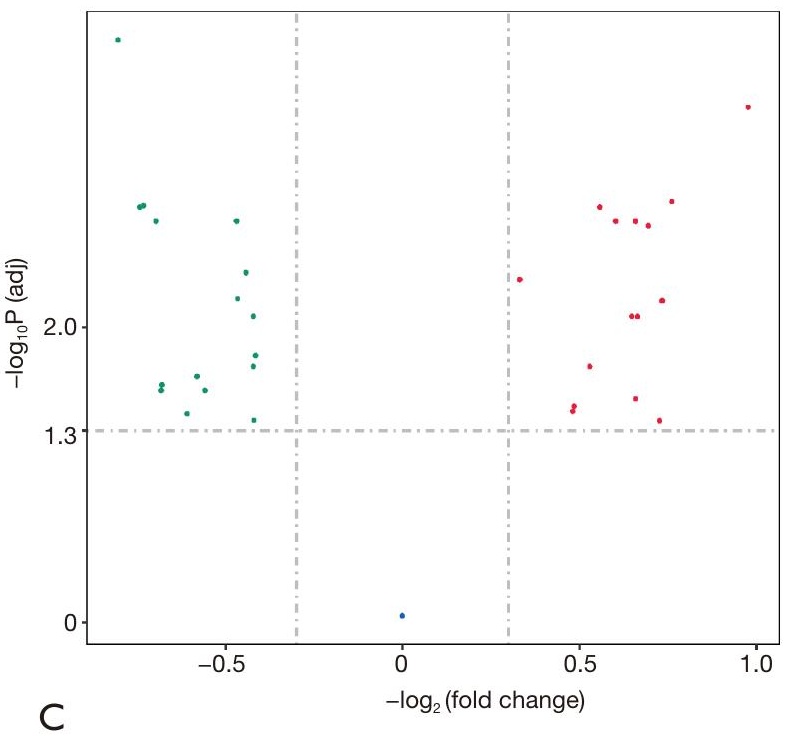

GO-CC

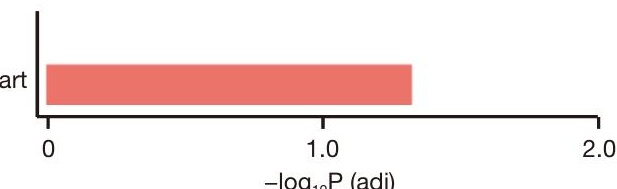

$E$

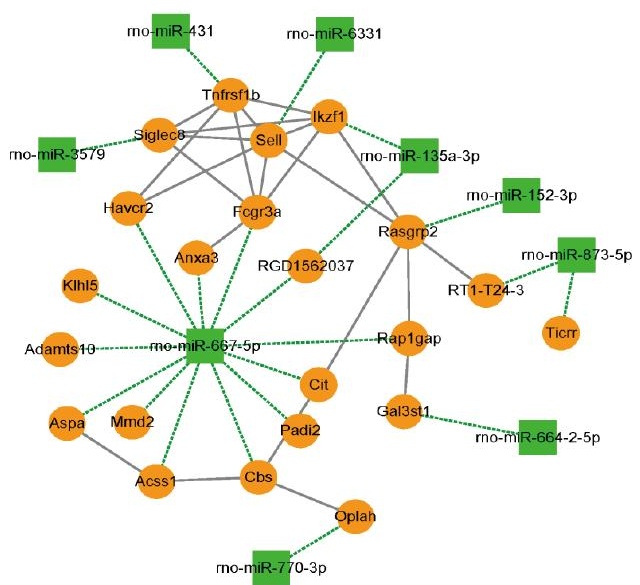

Figure 3 Bioinformatics analysis of the differentially expressed miRNAs and their target genes. (A) Heat map of differentially expressed miRNAs after DEX administration (red represents up-regulated miRNAs, and green represents down-regulated miRNAs); (B) volcano plot of differentially expressed miRNAs after DEX administration (red represents up-regulated miRNAs, and green represents down-regulated miRNAs); (C) GO analysis of the down-regulated miRNAs; (D) GO analysis of the target genes of down-regulated miRNAs; (E) PPI network and miRNA/mRNA integrated network analysis of down-regulated miRNAs and selected target genes. Orange color nodes indicate up-regulation and green color nodes indicate down-regulation. The size of the nodes represents the number of interactions. The solid line represents the interaction between genes and the dotted line represents the interaction between miRNAs and their target genes. DEX, dexmedetomidine; GO, Gene Ontology, BP, biological processes; CC, cellular components; PPI, protein-protein interaction. 
A

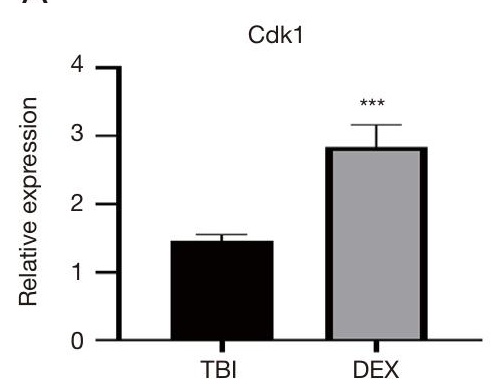

C

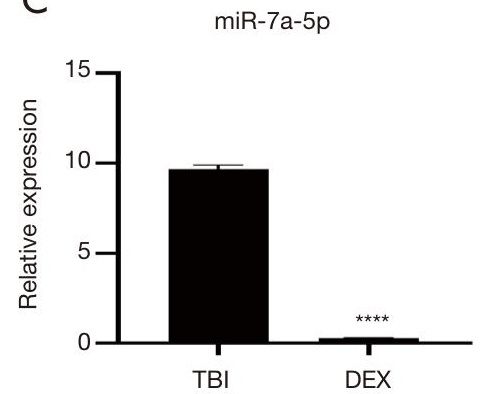

B
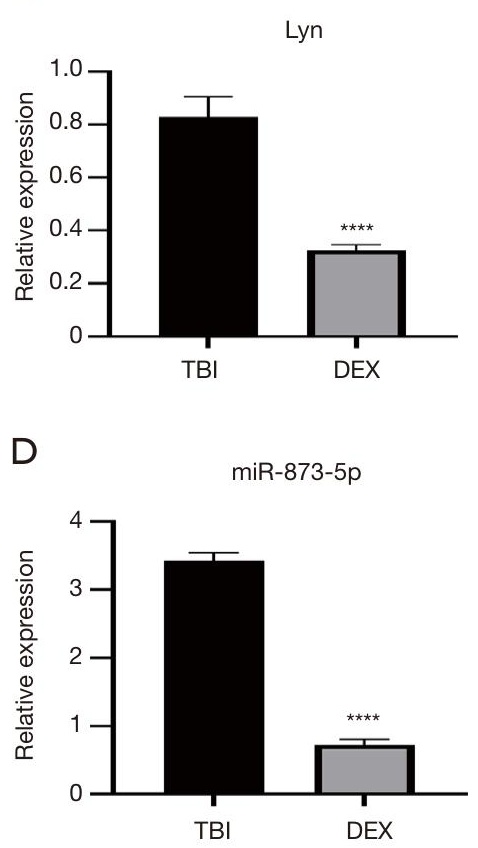

$\mathrm{E}$

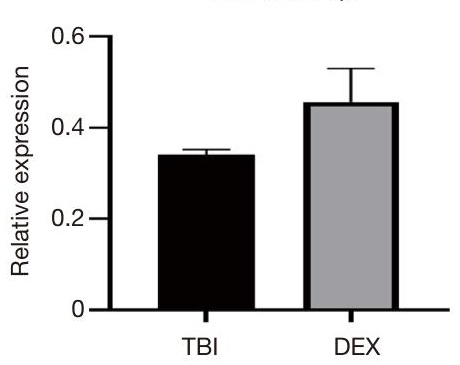

Figure 4 Validation results of qRT-PCR. (A-E) Expression levels of CDK1, Lyn, miR-7a-5p, miR-873-5p, and miR-135a-3p, respectively, relative to glyceraldehyde-3-phosphate dehydrogenase (GAPDH). ${ }^{* *} \mathrm{P}<0.001$ vs. TBI, ${ }^{* * * *} \mathrm{P}<0.0001$ vs. TBI. $\mathrm{N}=5$ in each group. TBI, traumatic brain injury; DEX, dexmedetomidine.

most of which [352] were up-regulated, and 35 deregulated miRNAs (18 up-regulated and 17 down-regulated). Through bioinformatics analyses with these 352 mRNAs up-regulated after DEX administration, multiple GO terms were discovered to be associated with the neuroprotective effect of DEX, including intercellular signal transduction, microtubule-based movement, and cytoskeletal protein binding. Cell adhesion molecules, which have been reported to play a crucial role in neuroprotection against TBI (31), were also detected in our study. The neural cell adhesion molecule (NCAM), a member of the adhesion molecule superfamily, is considered to be crucial for the development and maintenance of the central nervous system (42), and the results of the present study are consistent with this. Furthermore, in preclinical studies, bioactive peptides of NCAM have been used to successfully treat several neurological disorders, such as TBI, stroke, and Alzheimer's disease (43). However, whether there is an association between NCAM and DEX-induced neuroprotection, currently remains unclear and further research is required. Our results indicate that NCAM may be a potential novel target of DEX's neuroprotective effect against TBI.

In our bioinformatics analysis of up-regulated mRNAs,
25 hub genes were identified, among them, $L y n$ and $C d k 1$ were of particular interest. Lyn, a tyrosine kinase that belongs to the Src family, is reported to act as a key regulator of the B-cell signaling pathway (32), and might play an important role in DEX-induced neuroprotection. Since it has been reported that Lyn-ERK1/2-CREB activation attenuated rat brain ischemic damage via the up-regulation of brain-derived neurotrophic factor (BDNF) (44), the up-regulation of Lyn by DEX in this study might also contribute to the neuroprotective effect. The role of $L y n$ was further validated by qRT-PCR. Additionally, $C d k 1$, a promiscuous serine/threonine kinase that has been shown to phosphorylate a wide range of substrates during mitosis $(45,46)$, might be a crucial gene through which DEX exerts its neuroprotective effect. Indeed, besides their role in cellcycle control, several cyclin-dependent kinases (Cdks) have been reported to regulate ischemic neuronal death (47), and to be involved in neurodegenerative diseases (48). For example, $\mathrm{Cdk} 5$ inhibitor offers protection against neuronal death in Alzheimer's disease (AD) (49). Although Marlier et al. reported that inhibition of $C d k 1$ using genetic or pharmacological methods can achieve a neuroprotective effect against ischemic neuronal death (50), another 
study reached the opposite conclusion, in a lidocaineinduced cytotoxicity model, DEX was found to exert a neuroprotective effect consistent with the up-regulation of $C d k 1$ (51). For these two opposite results, we think the possible reasons are: (I) the neuron injury models in the two studies are different, (II) the principal ways that mediate neuronal death in diseases models above remain diverse. In our study, DEX's neuroprotective effect was associated with the up-regulation of $C d k 1$. Furthermore, we verified the up-regulation of $L y n$ and $C d k 1$ by qRT-PCR, and found that $C d k 1$ was stably up-regulated by $\mathrm{DEX}$, suggesting $C d k 1$ may be a potential target of DEX. Due to the complexity and heterogeneity of neuronal death, the same factor may play different roles in different disease models and processes, the exact of Cdk1 in TBI remains to be further elucidated. Furthermore, in the PPI network analysis, we found that Racgap1, a member of GAP family, was the hub gene with the most degrees; however, we failed to find any literature that can prove the relationship between Racgap1 and neurological disorders, and the biological function of Racgap1 in neurological diseases needs to be further studied and explored.

We performed functional enrichment analysis and miRNA/mRNA integrated analysis of nine down-regulated miRNAs and their target genes. Although numerous GO terms, including "negative regulation of small GTPasemediated signal transduction”, "Goli apparatus", "glycolipid biosynthetic process", "membrane lipid biosynthetic process" were detected; however, we failed to identify any KEGG pathway of significance. For the up-regulated miRNAs, we finally selected a very small number of target genes, and no significant result was obtained from the bioinformatics analyses. Therefore, we speculated that the up-regulated miRNAs were unlikely to be associated with DEX-induced neuroprotection. We also validated the expression levels of the top three down-regulated miRNAs, and the expression levels of two of them were found to be consistent with the results of RNA sequencing, and this result greatly encouraged us, as these results are expected to provide a good target for our follow-up research.

This study has some limitations and deficiencies. Firstly, only one dose of DEX $(100 \mu \mathrm{g} / \mathrm{kg})$ was administered intraperitoneally in 24 hours. The experimental protocol of this study was determined based on our previous studies, which proved that DEX administration can activate and enhance neuroprotective signaling in a TBI animal model (25). However, diverse, and even conflicting findings, might be acquired if rats are treated with different doses of DEX or if samples are harvested at different time points. Secondly, although we speculated and initially verified several pivotal genes and signaling pathways that may be associated with the neuroprotective effect of DEX against TBI, we did not confirm the specific mode by which these genes and pathways function, and the intrinsic connections or causal relationships between these genes and pathways remain to be explored. Finally, because of the small number of animals enrolled in this study, further validation by qRT-PCR was needed. It is necessary to conduct more gene expression evaluation studies focusing on the cellular signaling level or using different treatment times.

Based on RNA sequencing and bioinformatics analysis, this study investigated DEX-induced neuroprotection, and revealed deregulated mRNAs and miRNAs after drug administration. We identified and initially verified possible candidate genes and pathways that may be important for DEX's neuroprotective effect. Our study lays a foundation for follow-up studies, helps to clarify the mechanism of DEX, and provides new potential targets for the research and development of neuroprotective agents.

\section{Conclusions}

This study identified differentially expressed mRNAs and miRNAs following the administration of DEX in a TBI rat model. Bioinformatics analysis suggested that the B-cell receptor signaling and cell cycle pathways might involve in DEX-induced neuroprotection, Lyn and Cdk1 might be hub genes. Furthermore, qRT-PCR confirmed that the up-regulation of $C d k 1$, as well as the down-regulation of miR-7a-5p and miR-873-5p are associated with DEX's neuroprotective effect.

\section{Acknowledgments}

Funding: This study was supported by the National Natural Science Foundation of China (No. 81960817; 82060241), the Association Foundation Program of Yunnan Science and Technology Department and Kunming Medical University (2018FE001[-004]), the Innovation Team of Yunnan Province (2019HC014), and the Doctoral Innovation Fund of Kunming Medical University (2020D012).

\section{Footnote}

Reporting Checklist: The authors have completed the ARRIVE reporting checklist. Available at http://dx.doi. 
org/10.21037/apm-20-2346

Data Sharing Statement: Available at http://dx.doi. org/10.21037/apm-20-2346

Conflicts of Interest: All authors have completed the ICMJE uniform disclosure form (available at http://dx.doi. org/10.21037/apm-20-2346). The authors have no conflicts of interest to declare.

Ethical Statement: The authors are accountable for all aspects of the work in ensuring that questions related to the accuracy or integrity of any part of the work are appropriately investigated and resolved. Animal experiments were approved by Ethics Review Committee for Laboratory Animals of Kunming Medical University (Approval No. KMMU2020161). Animals were treated in accordance with Guide for the Care and Use of Laboratory Animals (8th edition, National Academies Press).

Open Access Statement: This is an Open Access article distributed in accordance with the Creative Commons Attribution-NonCommercial-NoDerivs 4.0 International License (CC BY-NC-ND 4.0), which permits the noncommercial replication and distribution of the article with the strict proviso that no changes or edits are made and the original work is properly cited (including links to both the formal publication through the relevant DOI and the license). See: https://creativecommons.org/licenses/by-nc-nd/4.0/.

\section{References}

1. Maas AIR, Menon DK, Adelson PD, et al. Traumatic brain injury: integrated approaches to improve prevention, clinical care, and research. Lancet Neurol 2017;16:987-48.

2. Hyder AA, Wunderlichb CA, Puvanachandraa P, et al. The impact of trumatic brain injury: a global perspective. NeuroRehabilitation 2007;22:341-53.

3. Wang P, Gao B, Wang M, et al. Challenges in the nursing care of intracranial carbapenem-resistant Escherichia coli infection after severe traumatic brain injury: a case report. Ann Palliat Med 2020;9:2381-5.

4. Guedes VA, Devoto C, Leete J, et al. Extracellular Vesicle Proteins and MicroRNAs as Biomarkers for Traumatic Brain Injury. Front Neurol 2020;11:663.

5. Mckee AC, Daneshvar DH. The neuropathology of traumatic brain injury. Handb Clin Neurol 2015;127:45-66.

6. Blennow K, Hardy J, Zetterberg H. The Neuropathology and Neurobiology of Traumatic Brain Injury. Neuron 2012;76:886-99.

7. Bao N, Tang B. Organ-Protective Effects and the Underlying Mechanism of Dexmedetomidine. Mediators Inflamm 2020;2020:6136105.

8. Liu H, Davis JR, Wu ZL, et al. Dexmedetomidine Attenuates Lipopolysaccharide Induced MCP-1 Expression in Primary Astrocyte. Biomed Res Int 2017;2017:6352159.

9. Liu Z, Wang Y, Wang Y, et al. Dexmedetomidine attenuates inflammatory reaction in the lung tissues of septic mice by activating cholinergic anti-inflammatory pathway. Int Immunopharmacol 2016;35:210-6.

10. Ning Q, Liu Z, Wang X, et al. Neurodegenerative changes and neuroapoptosis induced by systemic lipopolysaccharide administration are reversed by dexmedetomidine treatment in mice. Neurol Res 2017;39:357-66.

11. Endesfelder S, Makki H, von Haefen C, et al. Neuroprotective effects of dexmedetomidine against hyperoxia-induced injury in the developing rat brain. PLoS One 2017;12:e171498.

12. Sifringer $M$, von Haefen $C$, Krain $M$, et al. Neuroprotective effect of dexmedetomidine on hyperoxiainduced toxicity in the neonatal rat brain. Oxid Med Cell Longev 2015;2015:530371

13. Zhu YJ, Peng K, Meng XW, et al. Attenuation of neuroinflammation by dexmedetomidine is associated with activation of a cholinergic anti-inflammatory pathway in a rat tibial fracture model. Brain Res 2016;1644:1-8.

14. Duan X, Li Y, Zhou C, et al. Dexmedetomidine provides neuroprotection: impact on ketamine-induced neuroapoptosis in the developing rat brain. Acta Anaesthesiol Scand 2014;58:1121-6.

15. Lv J, Wei Y, Chen Y, et al. Dexmedetomidine attenuates propofol-induce neuroapoptosis partly via the activation of the PI3k/Akt/GSK3beta pathway in the hippocampus of neonatal rats. Environ Toxicol Pharmacol 2017;52:121-8.

16. Qian XL, Zhang W, Liu MZ, et al. Dexmedetomidine improves early postoperative cognitive dysfunction in aged mice. Eur J Pharmacol 2015;746:206-12.

17. Schoeler M, Loetscher PD, Rossaint R, et al. Dexmedetomidine is neuroprotective in an in vitro model for traumatic brain injury. BMC Neurol 2012;12:20.

18. Shen $M$, Wang S, Wen X, et al. Dexmedetomidine exerts neuroprotective effect via the activation of the PI3K/Akt/ mTOR signaling pathway in rats with traumatic brain injury. Biomed Pharmacother 2017;95:885-93.

19. Myserlis P, Radmanesh F, Anderson CD. Translational Genomics in Neurocritical Care: a Review. 
Neurotherapeutics 2020;17:563-80.

20. Izzy S, Liu Q, Fang Z, et al. Time-Dependent Changes in Microglia Transcriptional Networks Following Traumatic Brain Injury. Front Cell Neurosci 2019;13:307.

21. Lipponen A, Paananen J, Puhakka N, et al. Analysis of Post-Traumatic Brain Injury Gene Expression Signature Reveals Tubulins, Nfe212, Nfkb, Cd44 and S100a4 as Treatment Targets. Sci Rep 2016;6:31570.

22. Tang B, Pan Z, Yin K, et al. Recent Advances of Deep Learning in Bioinformatics and Computational Biology. Front Genet 2019;10:214.

23. Feeney DM, Boyeson MG, Linn RT, et al. Responses to cortical injury: methodology and local effects of contusions in the rat. Brain Res 1981;211:67-77.

24. Meng XE, Zhang Y, Li N, et al. Hyperbaric Oxygen Alleviates Secondary Brain Injury After Trauma Through Inhibition of TLR4/NF-kappaB Signaling Pathway. Med Sci Monit 2016;22:284-8.

25. Xiaoxiu Z, Haiying W, Yanxue W, et al. Dexmedetomidine exrts antioxidant effects on traumatic brain injury through activating Nrf2-ARE signaling pathway. Chinese Electronic Journal of Critical Care Medicine 2018;4:176-81.

26. Dweep H, Gretz N. miRWalk2.0: a comprehensive atlas of microRNA-target interactions. Nat Methods 2015;12:697.

27. Huang DW, Sherman BT, Tan Q, et al. The DAVID Gene Functional Classification Tool: a novel biological modulecentric algorithm to functionally analyze large gene lists. Genome Biol 2007;8:R183.

28. Szklarczyk D, Franceschini A, Wyder S, et al. String v10 protein protein interaction networks integrated over the tree of life. Nucleic Acids Res 2015;43:D447-52.

29. Cline MS, Smoot M, Cerami E, et al. Integration of biological networks and gene expression data using Cytoscape. Nat Protoc 2007;2:2366-82.

30. Li F, Wang X, Deng Z, et al. Dexmedetomidine reduces oxidative stress and provides neuroprotection in a model of traumatic brain injury via the PGC-1alpha signaling pathway. Neuropeptides 2018;72:58-64.

31. Hemphill MA, Dauth S, Yu CJ, et al. Traumatic brain injury and the neuronal microenvironment: a potential role for neuropathological mechanotransduction. Neuron 2015;85:1177-92.

32. Brodie EJ, Infantino S, Low MSY, et al. Lyn, Lupus, and (B) Lymphocytes, a Lesson on the Critical Balance of Kinase Signaling in Immunity. Front Immunol 2018;9:401.

33. Jones MC, Zha J, Humphries MJ. Connections between the cell cycle, cell adhesion and the cytoskeleton. Philos Trans R Soc Lond B Biol Sci 2019;374:20180227.
34. Lin N, Vutskits L, Bebawy JF, et al. Perspectives on Dexmedetomidine Use for Neurosurgical Patients. J Neurosurg Anesthesiol 2019;31:366-77.

35. Sun Z, Zhao T, Lv S, et al. Dexmedetomidine attenuates spinal cord ischemia-reperfusion injury through both antiinflammation and anti-apoptosis mechanisms in rabbits. J Transl Med 2018;16:209.

36. Sun YB, Zhao H, Mu DL, et al. Dexmedetomidine inhibits astrocyte pyroptosis and subsequently protects the brain in in vitro and in vivo models of sepsis. Cell Death Dis 2019;10:167.

37. Afshordel S, Kern B, Clasohm J, et al. Lovastatin and perillyl alcohol inhibit glioma cell invasion, migration, and proliferation--impact of Ras-/Rho-prenylation. Pharmacol Res 2015;91:69-77.

38. Diaz-Arrastia R, Kochanek PM, Bergold P, et al. Pharmacotherapy of traumatic brain injury: state of the science and the road forward: report of the Department of Defense Neurotrauma Pharmacology Workgroup. J Neurotrauma 2014;31:135-58.

39. Dougall D, Poole N, Agrawal N. Pharmacotherapy for chronic cognitive impairment in traumatic brain injury. Cochrane Database Syst Rev 2015;(12):CD009221.

40. Alam A, Suen KC, Hana Z, et al. Neuroprotection and neurotoxicity in the developing brain: an update on the effects of dexmedetomidine and xenon. Neurotoxicol Teratol 2017;60:102-16.

41. Jiang $\mathrm{L}, \mathrm{Hu} \mathrm{M}, \mathrm{Lu} \mathrm{Y}$, et al. The protective effects of dexmedetomidine on ischemic brain injury: A metaanalysis. J Clin Anesth 2017;40:25-32.

42. Li S, Bock E, Berezin V. Neuritogenic and neuroprotective properties of peptide agonists of the fibroblast growth factor receptor. Int J Mol Sci 2010;11:2291-305.

43. Chu C, Gao Y, Lan X, et al. NCAM Mimetic Peptides: Potential Therapeutic Target for Neurological Disorders. Neurochem Res 2018;43:1714-22.

44. Zhang QG, Han D, Hu SQ, et al. Positive modulation of AMPA receptors prevents downregulation of GluR2 expression and activates the Lyn-ERK1/2-CREB signaling in rat brain ischemia. Hippocampus 2010;20:65-77.

45. Blethrow JD, Glavy JS, Morgan DO, et al. Covalent capture of kinase specific phosphopeptides reveals Cdk1cyclin B substrates. Proc Natl Acad Sci 2008;105:1442.

46. Malumbres M. Cyclin-dependent kinases. Genome Biol 2014;15:122.

47. Rashidian J, Iyirhiaro GO, Park DS. Cell cycle machinery and stroke. Biochim Biophys Acta 2007;1772:484-93.

48. Frade JM, Ovejero-Benito MC. Neuronal cell cycle: 
the neuron itself and its circumstances. Cell Cycle 2015;14:712-20.

49. Cortés N, Guzmán-Martínez L, Andrade V, et al. CDK5: A Unique CDK and Its Multiple Roles in the Nervous System. J Alzheimers Dis 2019;68:843-55.

50. Marlier Q, Jibassia F, Verteneuil S, et al. Genetic and pharmacological inhibition of $\mathrm{Cdk} 1$ provides neuroprotection towards ischemic neuronal death. Cell

Cite this article as: Yang $\mathrm{L}, \mathrm{Wu} \mathrm{H}$, Yang F, Li P, Huang Y, Zhang X, Qian C. Identification of candidate genes and pathways in dexmedetomidine-induced neuroprotection in rats using RNA sequencing and bioinformatics analysis. Ann Palliat Med 2021;10(1):372-384. doi: 10.21037/apm-20-2346
Death Discov 2018;4:43.

51. Tan Y, Bi X, Wang Q, et al. Dexmedetomidine protects PC12 cells from lidocaine-induced cytotoxicity via downregulation of Stathmin 1. Drug Des Devel Ther 2019;13:2067-79.

(English Language Editor: J. Reynolds) 


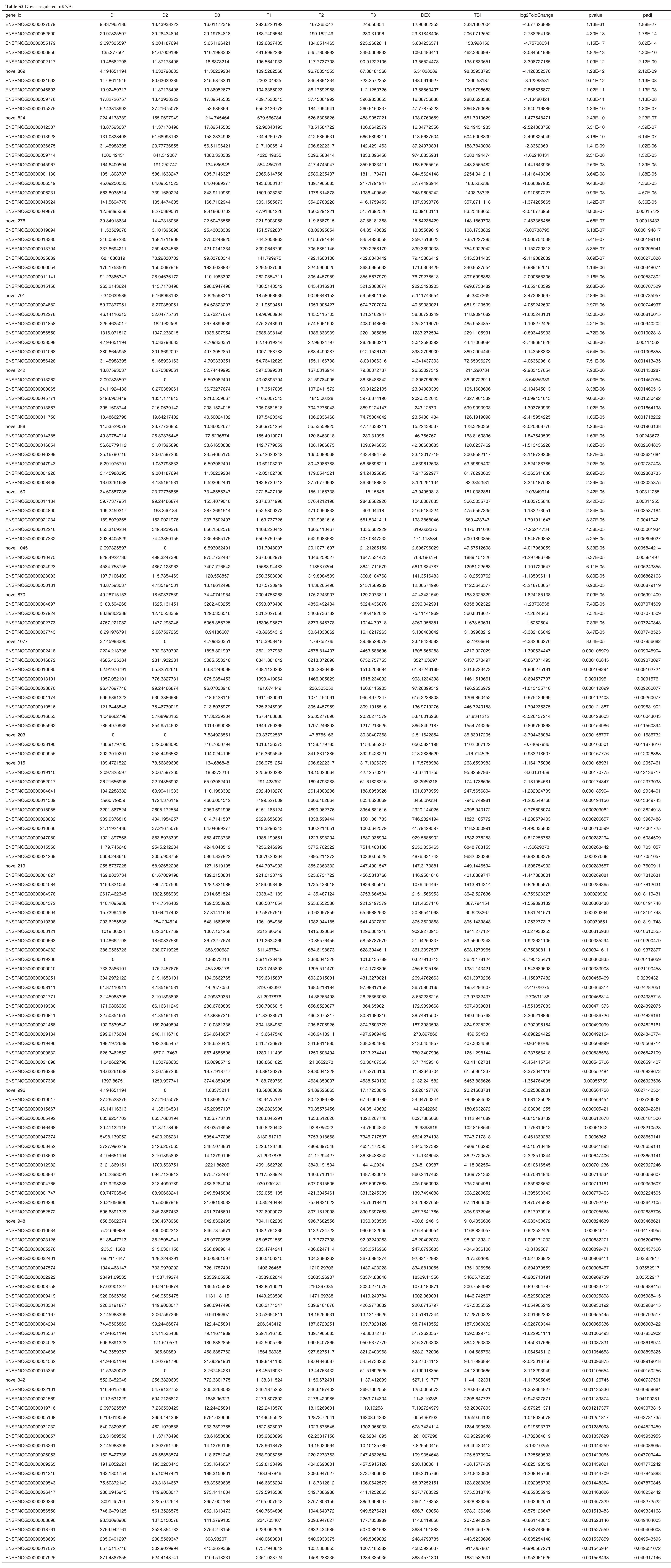


Table S3 Up-regulated miRNAs

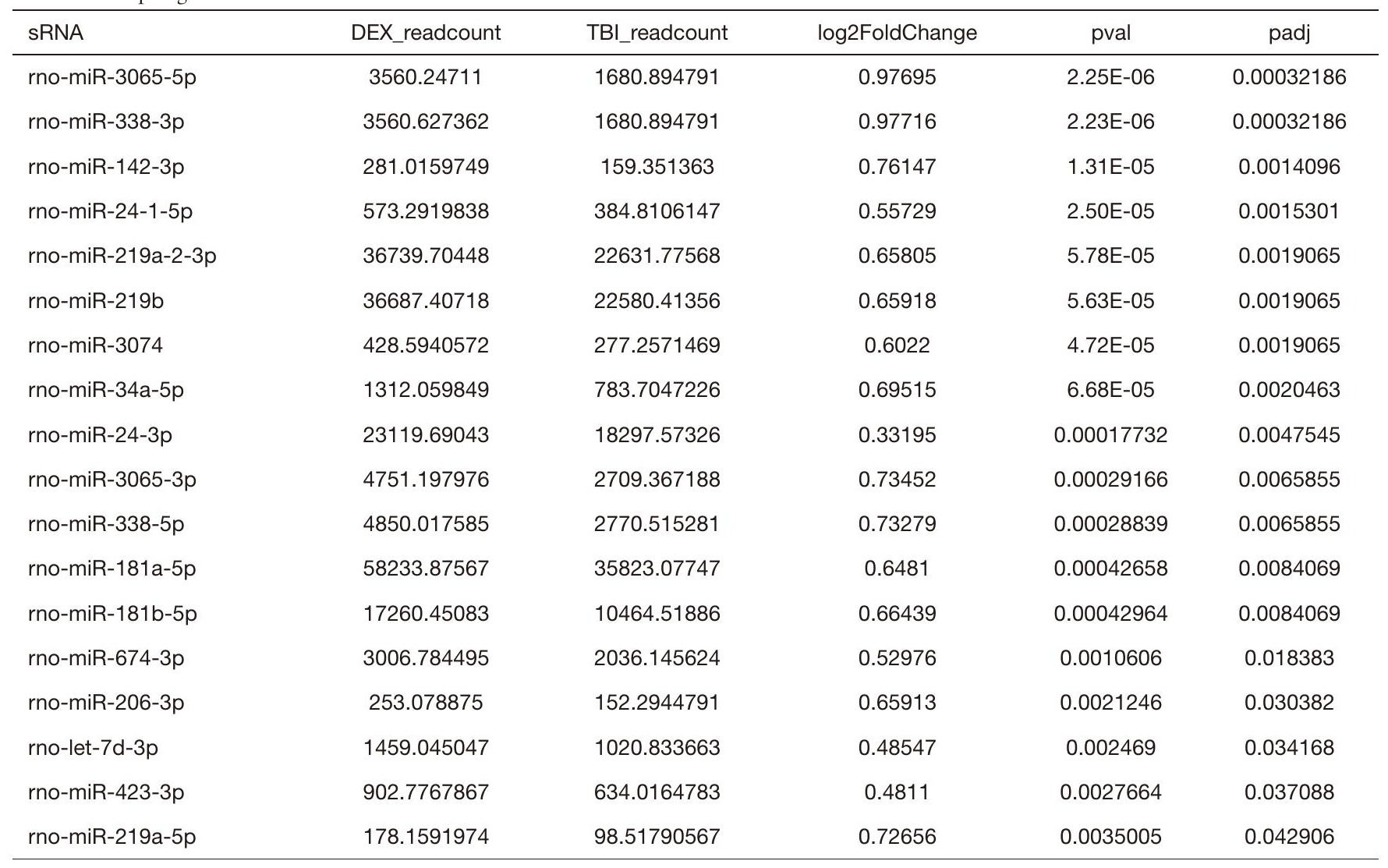


Table S4 Down-regulated miRNAs

\begin{tabular}{|c|c|c|c|c|c|}
\hline sRNA & DEX_readcount & TBI_readcount & log2FoldChange & pval & padj \\
\hline rno-miR-873-5p & 941.1774494 & 1618.701615 & -0.73274 & $1.75 \mathrm{E}-05$ & 0.0015006 \\
\hline rno-miR-135a-3p & 217.3176405 & 378.9180432 & -0.74337 & $2.14 \mathrm{E}-05$ & 0.0015301 \\
\hline rno-miR-3579 & 2004.125885 & 2799.341916 & -0.46856 & 4.09E-05 & 0.0019065 \\
\hline rno-miR-7a-2-3p & 304.5941613 & 511.4763462 & -0.69608 & $5.38 \mathrm{E}-05$ & 0.0019065 \\
\hline rno-miR-6331 & 885.9687917 & 1214.636031 & -0.44204 & 0.00014881 & 0.0042559 \\
\hline rno-miR-539-3p & 834.6599286 & 1166.582415 & -0.46587 & 0.0002534 & 0.0063947 \\
\hline rno-miR-379-5p & 51224.19295 & 69256.1727 & -0.42184 & 0.00043112 & 0.0084069 \\
\hline rno-miR-7b & 42416.92922 & 65476.64811 & -0.58089 & 0.001309 & 0.021599 \\
\hline rno-miR-152-3p & 2306.436134 & 3910.824602 & -0.6804 & 0.001544 & 0.024533 \\
\hline rno-miR-488-5p & 44.05245606 & 75.19996613 & -0.68234 & 0.0018047 & 0.026698 \\
\hline rno-miR-667-5p & 132.3334002 & 200.2719997 & -0.55831 & 0.0017463 & 0.026698 \\
\hline rno-miR-873-3p & 284.8529718 & 454.0614683 & -0.60966 & 0.002957 & 0.038442 \\
\hline rno-miR-664-2-5p & 213.6551185 & 288.7347305 & -0.41984 & 0.0033801 & 0.042649 \\
\hline
\end{tabular}




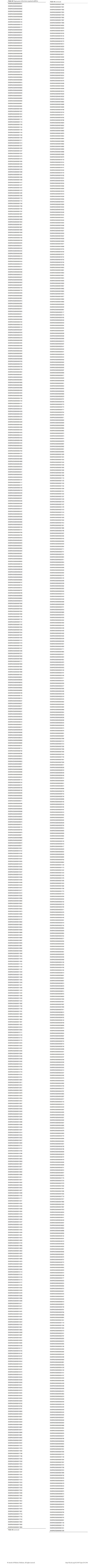


Table S7 Selected target genes of the up-regulated miRNAs

\begin{tabular}{lcc}
\hline sRNA_id & mRNA_id & mRNA_name \\
\hline rno-miR-142-3p & ENSRNOG00000005108 & Wfs1 \\
rno-miR-34a-5p & ENSRNOG00000056558 & Gabra3 \\
rno-miR-24-3p & ENSRNOG00000011858 & Unc5d \\
rno-miR-3065-3p & ENSRNOG00000010516 & Plau \\
rno-miR-3065-3p & ENSRNOG00000018384 & Adam12 \\
rno-miR-674-3p & ENSRNOG00000010841 & Col8a2 \\
\hline
\end{tabular}

Table S8 Selected target genes of the down-regulated miRNAs

\begin{tabular}{|c|c|c|}
\hline sRNA_id & mRNA_id & mRNAname \\
\hline rno-miR-873-5p & ENSRNOG00000045924 & RT1-T24-3 \\
\hline rno-miR-135a-3p & ENSRNOG00000004444 & Ikzf1 \\
\hline rno-miR-135a-3p & ENSRNOG00000027938 & RGD1562037 \\
\hline rno-miR-6331 & ENSRNOG00000002776 & Sell \\
\hline rno-miR-431 & ENSRNOG00000016575 & Tnfrsf1b \\
\hline rno-miR-770-3p & ENSRNOG00000011781 & Oplah \\
\hline rno-miR-152-3p & ENSRNOG00000021098 & Rasgrp2 \\
\hline rno-miR-667-5p & ENSRNOG00000002045 & Anxa3 \\
\hline rno-miR-667-5p & ENSRNOG00000007102 & Acss 1 \\
\hline rno-miR-667-5p & ENSRNOG00000007574 & Padi2 \\
\hline rno-miR-667-5p & ENSRNOG00000008421 & Klhl5 \\
\hline rno-miR-667-5p & ENSRNOG00000008857 & Adamts10 \\
\hline rno-miR-667-5p & ENSRNOG00000013825 & Rap1gap \\
\hline rno-miR-667-5p & ENSRNOG00000019659 & Aspa \\
\hline rno-miR-667-5p & ENSRNOG00000024382 & Fcgr3a \\
\hline
\end{tabular}

